\title{
Cisto ósseo traumático em área incomum: relato de caso
}

Traumatic bone cyst in an unusual area: case report

Quiste óseo traumático en un área inusual: reporte de caso

Mirela Caroline SILVA ${ }^{1}$

Tiburtino José de LIMA-NETO ${ }^{1}$

Anderson Maikon de Souza SANTOS ${ }^{1}$

Leonardo Perez FAVERANI ${ }^{1}$

Sirius Dan INAOKA ${ }^{2}$

Davi Felipe Neves DA COSTA ${ }^{2}$

${ }^{1}$ Departamento de Cirurgia e Traumatologia Bucomaxilofacial e Clínica Integrada, Faculdade de Odontologia, UNESP Univ. Estadual Paulista, 16015-050 Araçatuba - SP, Brasil

${ }^{2}$ Serviço de Residência em Cirurgia e Traumatologia Bucomaxilofacial do Hospital Universitário Lauro Wanderley, UFPB Universidade Federal da Paraíba, 58059-900 João Pessoa - PB, Brasil

\section{Resumo}

Introdução: O cisto ósseo traumático possui diversas nomenclaturas e é classificado como um pseudocisto, por não apresentar cápsula e revestimento epitelial. Acomete principalmente pacientes jovens na região posterior de mandíbula, podendo este ter histórico de trauma. Por serem assintomáticos, muitas vezes são diagnosticados em exames radiográficos de rotina. A exploração da cavidade é indicada tanto para diagnóstico, após analise histopatológica, quanto para terapêutica. Objetivo: O objetivo desse trabalho é apresentar as características de cisto ósseo traumático para diagnostico e tratamento, a partir do relato de um caso de dois cistos ósseos em regiões de menor incidência na mandíbula. Relato de caso: Paciente de 15 anos foi diagnosticado com duas lesões de cisto ósseo traumático, após uma radiografia de rotina para tratamento ortodôntico. As lesões eram de aproximadamente $10 \mathrm{~mm}$ sendo duas cavidades no mento e de $15 \mathrm{~mm}$ na região do côndilo esquerdo e assintomáticas. Para o diagnóstico e tratamento, foi realizado uma aspiração na lesão do mento e posteriormente exploração cirúrgica em ambas lesões; e mesmo observando certa escassez de tecido para a exploração, uniu-se o exame com as características clinicas do paciente e obteve-se um diagnóstico definitivo de cisto ósseo traumático. $O$ mesmo passou por acompanhamento clínico-radiográfico e pode ser observada regressão da lesão. Conclusão: Se faz importante o conhecimento das características dessa lesão para correto diagnóstico e tratamento, mesmo que o tratamento seja feito somente com exploração cirúrgica e proservação.

Descritores: Cistos Ósseos; Diagnóstico; Radiografia.

\section{Abstract}

Introduction: The traumatic bone cyst has several nomenclatures and is classified as a pseudocyst, as it does not have an epithelial capsule and lining. It mainly affects young patients in the posterior region of the mandible, which may have a history of trauma. Because they are asymptomatic, they are often diagnosed on routine radiographic examinations. The exploration of the cavity is indicated both for diagnosis, after histopathological analysis, and for therapy. Objective: The objective of this study is to present the characteristics of a traumatic bone cyst for diagnosis and treatment, based on the report of a case of two bone cysts in regions of lower incidence in the mandible. Case report: A 15-year-old patient was diagnosed with two traumatic bone cyst lesions after a routine radiography for orthodontic treatment. The lesions were approximately $10 \mathrm{~mm}$, two cavities in the chin and $15 \mathrm{~mm}$ in the left condyle and both asymptomatic. For diagnosis and treatment, an aspiration was performed on the chin injury and later surgical exploration on both injuries; and even observing a certain scarcity of tissue for exploration, the examination was combined with the patient's clinical characteristics and a definitive diagnosis of traumatic bone cyst was obtained. The patient underwent clinical and radiographic monitoring and regression of the lesion can be observed. Conclusion: Knowledge of the characteristics of this lesion is important for correct diagnosis and treatment, even if the treatment is done only with surgical exploration and proservation.

Descriptors: Bone Cysts; Diagnosis; Radiography.

\section{Resumen}

Introducción: el quiste óseo traumático tiene várias nomenclaturas y se clasifica como un seudoquiste, ya que no tiene una cápsula y revestimiento epiteliales. Afecta principalmente a pacientes jóvenes en la región posterior de la mandíbula, que pueden tener antecedentes de trauma. Debido a que son asintomáticos, a menudo se diagnostican en exámenes radiográficos de rutina. La exploración de la cavidad está indicada tanto para el diagnóstico, después del análisis histopatológico, como para la terapia. Objetivo: El objetivo de este trabajo es presentar las características del quiste óseo traumático para el diagnóstico y el tratamiento, en base al informe de un caso de dos quistes óseos en regiones de menor incidencia en la mandíbula. Caso clínico: un paciente de 15 años fue diagnosticado con dos lesiones quísticas óseas traumáticas después de una radiografía de rutina para el tratamiento de ortodoncia. Las lesiones eran de aproximadamente $10 \mathrm{~mm}$, dos cavidades en el mentón y $15 \mathrm{~mm}$ en el cóndilo izquierdo y asintomáticas. Para el diagnóstico y el tratamiento, se realizó una aspiración en la lesión del mentón y luego exploración quirúrgica en ambas lesiones; e incluso observando una cierta escasez de tejido para exploración, el examen se combinó con las características clínicas del paciente y se obtuvo un diagnóstico definitivo de quiste óseo traumático. El paciente se sometió a monitorización clínica y radiográfica y se puede observar la regresión de la lesión. Conclusión: El conocimiento de las características de esta lesión es importante para un diagnóstico y tratamiento correctos, incluso si el tratamiento se realiza solo con exploración quirúrgica y conservación.

Descriptores: Quistes Óseos; Diagnóstico; Radiografía.

INTRODUÇÃO

Cisto ósseo traumático ou também denominado cisto ósseo simples, hemorrágico, solitário, idiopático e cavidade óssea idiopática é uma lesão benigna caracterizada pela presença de uma cavidade óssea que pode ser vazia ou preenchida por um conteúdo fluído sendo, em ambos os casos, livre de revestimento epitelial ${ }^{1-5}$. Devido a não possuírem características morfológicas de uma lesão cística como cápsula e revestimento epitelial, ele foi classificado pela Organização Mundial da Saúde (OMS) como um pseudocisto $^{3,4}$. Sua etiopatogenia ainda é desconhecida, porém existem algumas teorias 
que buscam explicar sua causa; a principal delas é a do trauma prévio no tecido ósseo. Acredita-se que o trauma leva a formação de um hematoma intraósseo, o que compromete o suprimento sanguíneo na área e leva a reabsorção óssea osteoclástica ${ }^{2,4}$.

Essa lesão é rara, mas acomete frequentemente a região posterior da mandíbula, principalmente no corpo ou na sínfise; especificamente na região entre 0 canino e o terceiro molar ${ }^{1,2,4}$. A maioria dos pacientes são jovens, entre a segunda década de vida, raras em crianças menores de 5 anos de idade 4. Clinicamente é assintomático e, por isso, frequentemente são diagnosticados em exames radiográficos de rotina; os dentes envolvidos na lesão apresentam vitalidade pulpar preservada ${ }^{2,4}$. Na radiografia ele se encontra como uma imagem radiolúcida unilocular com um contorno irregular, porém bem ou parcialmente definido; com ou sem revestimento esclerótico na periferia da lesão ${ }^{1}$, 2. Uma característica presente no cisto ósseo traumático é o "efeito escalonado" quando se estende entre as raízes dos dentes ${ }^{2}$.
Embora
suas
características

morfológicas não sejam iguais de um cisto, seu diagnostico diferencial somente é conseguido com exploração cirúrgica da cavidade devido a possibilidade de confusão com uma grande variedade de lesões radiolúcidas odontogênicas ou não odontogênicas ${ }^{4,5}$. O material para 0 exame na maioria das vezes é escasso ou inexistente, dependendo do conteúdo encontrado, o que dificulta ainda mais a obtenção de um diagnostico definitivo o qual deve ser baseado em todos os dados obtidos incluindo características clinicas, radiológicas e descobertas cirúrgicas. Pode-se realizar antes da exploração uma manobra chamada de Punção Aspirativa por Agulha Fina (PAAF) onde encontra-se na maioria das vezes nessas lesões um conteúdo liquido sero-sanguinolento 5. A exploração da cavidade, nesse caso, serve tanto como uma manobra para diagnostico quanto para terapia definitiva onde as paredes são curetadas para obtenção de material para exame histopatológico e para produção de sangramento na cavidade o que leva a uma obliteração da lesão e nova formação óssea ${ }^{2,4}$. Essa lesão normalmente se apresenta sem complicações e muitas vezes podem ter resoluções espontâneas, porém não é recomendável aguardar a regressão espontânea da lesão devido a possibilidade de erros diagnósticos, o que pode levar a complicações sérias ${ }^{1}$.

O objetivo do artigo é apresentar as características do cisto ósseo traumático para obtenção de um diagnostico definitivo efetivo e apresentar um relato de caso clínico que ocorreu sem complicações.

\section{CASO CLÍNICO}

Paciente de 15 anos do sexo masculino foi atendido no Hospital Universitário Lauro Wanderley (HULW - UFPB) encaminhado após realizar uma panorâmica de rotina para tratamento ortodôntico e constatar a presença duas lesões (Figura 1). A primeira delas era uma região radiolúcida, multilocular, de aproximadamente $10 \mathrm{~mm}$ e bem definida no mento, região periapical dos dentes 41,42 e 43 . Outra lesão pôde ser observada como uma região radiolúcida, unilocular, de aproximadamente $15 \mathrm{~mm}$ e bem definida, anterior e superior, na região de ramo se estendendo para colo do côndilo.

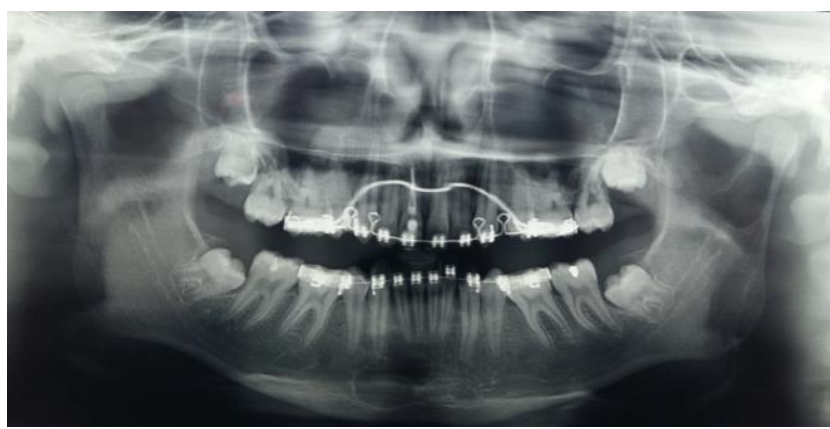

Figura 1: Radiografia panorâmica evidenciando duas lesões radiolúcidas nas regiões mentoniana e do ramo/colo mandibular.

O exame físico intra e extraoral não apresentaram alterações; o paciente também não relatava sintomas e nem relato de trauma prévio na região. Foi realizada um punção aspirativa com agulha fina (PAAF) na região do mento e do côndilo onde se obteve um liquido serosanguíneo (Figura 2).

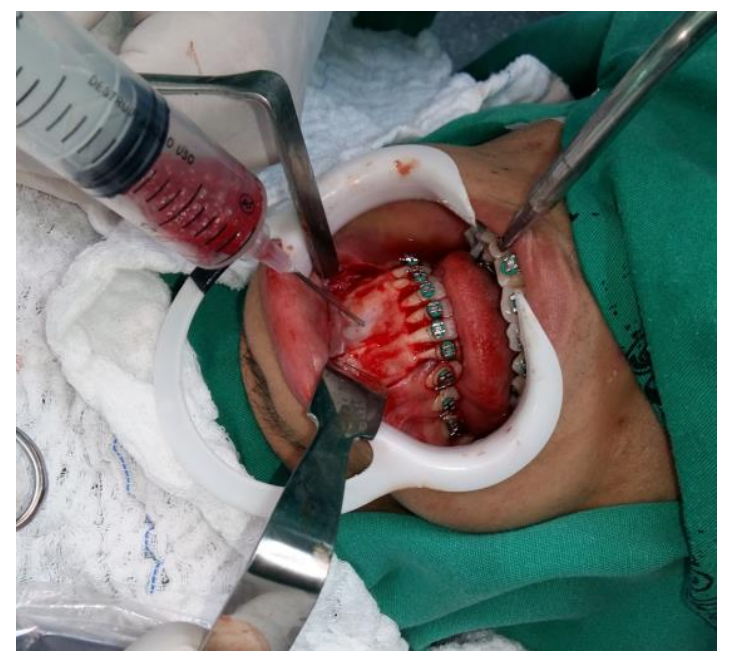

Figura 2: PAAF sendo realizado na região do mento. Obbserva-se conteúdo seosanguíneo no interior da seringa.

Posteriormente, foi realizada uma exploração cirúrgica para obtenção de conteúdo 
para exame histopatológico, já que a hipótese diagnostica era para um queratocisto (Figuras 3 e 4). Nesta exploração pode-se observar a ausência de revestimento epitelial e conteúdo escasso para o exame, porém este, juntamente com as características clínicas observadas, permitiu a obtenção de um diagnostico definitivo de cisto ósseo traumático. A cavidade foi curetada e preenchida com conteúdo sanguíneo e o paciente foi acompanhado clinicamente e com exames radiográficos até 0 desaparecimento da lesão e formação de tecido ósseo na região, o que ocorreu sem complicações.

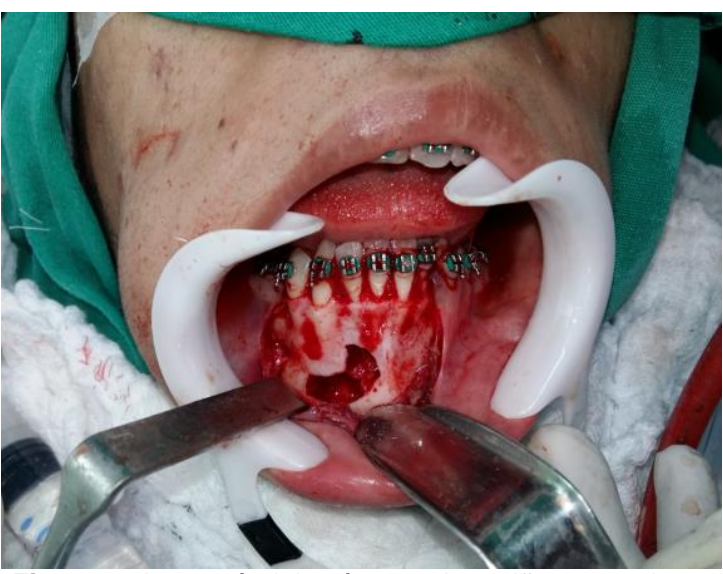

Figura 3: Acesso às lojas ósseas da região mentoniana.

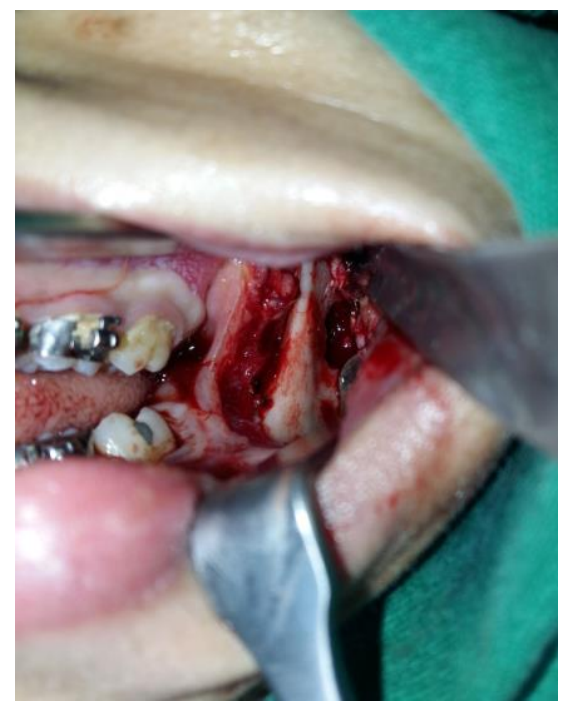

Figura 4: Acesso às lojas ósseas da região do colo mandibular.

DISCUSSÃO

O cisto ósseo simples é uma lesão rara que acomete principalmente pacientes jovens e quase exclusivamente a mandíbula. Por ser assintomático e, na maioria das vezes, não apresentarem alterações clinicas são descobertos em exames radiográficos de rotina $^{2,4}$. No caso apresentado, o paciente também não apresentava sintomas e alterações clínicas e as lesões foram descobertas em uma radiografia panorâmica realizada para tratamento ortodôntico. Mesmo que seja, na maioria das vezes, assintomático existem sintomas incomuns que podem acompanhar essa lesão, que incluem: sensibilidade dentária, parestesia, fístulas, erupção retardada dos dentes e fratura patológica da mandíbula. É necessário realizar o exame físico de forma cuidadosa, pois pode haver a presença de edema intra e extrabucal, mesmo que de forma rara, podendo causar até mesmo assimetria facial dependendo da extensão da lesão. Os dentes na região da lesão são vitais e sem mobilidade, deslocamento ou reabsorções de raízes; o que também é uma característica importante para o diagnostico definitivo 3 . No relato, não foi observado envolvimento dentário e alterações intra ou extraorais.

A avaliação radiográfica pode ser realizada através da panorâmica, o que é um ótimo meio para análise da lesão ou até mesmo através de tomografias computadorizadas ou ressonância magnética com intuito de descartar a necessidade de punção aspirativa prévia, pelo fato de possibilitar a confirmação da presença ou não de conteúdo fluído dentro da cavidade. Nos exames de imagem deve-se analisar expansão ou erosão da placa cortical; característica incomum, porém que pode estar presente nesse tipo de lesão ${ }^{3}$. Na radiografia panorâmica analisada no caso foi possível observar duas lesões semelhantes ao cisto ósseo traumático, sendo ambas radiolúcida e com limites bem definido; a da região do mento era multilocular e a da região do côndilo era unilocular, todas características semelhantes as encontradas na literatura que caracterizam 0 cisto ósseo traumático ${ }^{2,4}$.

Optou-se pela PAAF e inspeção cirúrgica como método de diagnóstico e tratamento, o que é indicado e realizado na maioria dos casos $^{2,4}$. Durante o procedimento foi possível observar um material escasso, porém foi possível realizar a curetagem e obtenção de material para o exame histopatológico. Este procedimento é preconizado como tratamento, pois o sangue devido ao procedimento cirúrgico oblitera rapidamente a lesão e permite a formação óssea ${ }^{4}$. Pode-se optar por um tratamento por fechamento por segunda intenção da ferida cirúrgica, juntamente com curativos com gaze furacinada com troca a cada 2 dias e irrigação da cavidade com soro fisiológico e bochechos com clorexidina $0,12 \%$; porém não foi esse tratamento utilizado no presente caso, devido a maior dificuldade e complexidade e maior necessidade de colaboração do paciente ${ }^{3}$. Além disso, o tratamento de escolha mostrou-se efetivo para o caso. 
O exame histopatológico confirmou 0 diagnostico de cisto ósseo traumático, assim como outros dados obtidos no atendimento como no exame físico e radiográfico. Dados os quais devem ser analisados e associados juntamente com a história clínica, exploração cirúrgica e também os resultados dos exames histopatológicos devido aos diagnósticos diferenciais dessa lesão como cisto dentígero, ceratocisto odontogênico, ameloblastoma e até mesmo uma associação com outra patologia como a púrpura trombocitopênica, por exemplo ${ }^{3}$. Devido a isso também não é recomendável aguardar a regressão espontânea da lesão, o que pode levar a sérias complicações ${ }^{1}$. No presente caso não houve complicações, assim como na maioria dos casos em que são realizados o diagnostico e os tratamentos adequados. O acompanhamento pós-operatório com controle radiográfico devem ser feitos de 4 a 7 meses, onde deve ser possível observar a formação de tecido ósseo e desaparecimento da lesão; o que também foi realizado nesse caso ${ }^{3}$.

CONCLUSÃO

É importante o conhecimento das características do cisto ósseo traumático para seu correto diagnostico e também associação com exames de imagens, intervenção cirúrgica e resultado do exame histopatológico para exclusão de outras lesões que possam ser semelhantes a ele. Alem disso é necessário tratamento correto para menor risco de ocorrência de complicações, pois mesmo que raras, se não diagnosticado de forma correta, elas podem existir.

\section{REFERÊNCIAS}

1. Palma VC, da Silva Caetano R, Borges $A H$, Volpato LER. Immediate Insertion of Dental Implants Immediate Insertion in Bilateral Traumatic Bone Cysts With or Without a Bone Grafting Material: A 5-Year Follow-Up Case Report. J Oral Implantol. 2019;45(4):297-299.

2. Xanthinaki AA, Choupis KI, Tosios K, Pagkalos VA, Papanikolaou SI. Traumatic bone cyst of the mandible of possible iatrogenic origin: a case report and brief review of the literature. Head Face Med. 2006;2:40.

3. Lago CA, Cauás M, Pereira AM, Portela L. Cisto ósseo traumático em mandíbula: relato de caso. Rev Cir Traumatol Buco-Maxilo-Fac. 2006;2(6):23-8.

4. Nunes AC, Kawata LT, Abreu FC, Pereira LJ, Takahama Junior A. Cisto ósseo simples: relato de dois casos e revisão de literatura. Arch Health Invest. 2012;1(1):11-7.
5. Alencar AKO, De Araújo Filho JCWP, Noleto JW, Hochulli-Vieira E, Garcia Júnior IR, Rocha JF. Aspectos técnicos de tratamento do cisto ósseo traumático: relato de caso. Rev Assoc Paul Cir Dent. 2015;69(1):26-9.

\section{CONFLITO DE INTERESSES}

Os autores declaram não haver conflitos de interesse

\section{AUTOR PARA CORRESPONDÊNCIA}

\section{Mirela Caroline Silva}

Avenida Joaquim Pompeu de Toledo, 1777, Condomínio Duetto Boulevard, apartamento 145A 16020-405 Araçatuba - SP,Brasil

Email: mirela_carol12@hotmail.com 